(C) 2019. This manuscript version is made available under the CC-BY-NC-ND 4.0 license. This is the Accepted Manuscript version of an article accepted for publication in JINST. IOP Publishing Ltd is not responsible for any errors or omissions in this version of the manuscript or any version derived from it. The Version of Record is available online at https://doi.org/10.1088/1748-0221/14/01/C01003 $20^{\text {TH }}$ Workshop on Radiation IMAGING Detectors

WHEN 24-28 JUNE 2018

WHERE SUNDSVALL, SWEDEN

\title{
Gain and Noise in GaAs/AIGaAs Avalanche Photodiodes with Thin Multiplication Regions
}

\author{
Camilla Nichetti $^{a, b, 1}$, Tereza Steinhartova ${ }^{b, c}$, Matias Antonelli ${ }^{a}$, Giuseppe Cautero ${ }^{a, d}$, Ralf \\ Hendrik Menk $^{a, d, e}$, Alessandro Pilotto ${ }^{f}$, Francesco Driussi ${ }^{f}$, Pierpaolo Palestri ${ }^{f}$, Luca \\ Selmi $^{g}$, Fulvia Arfelli $^{b, d}$, Giorgio Biasiol ${ }^{c}$ \\ ${ }^{a}$ Elettra-Sincrotrone Trieste S.C.p.A, Area Science Park Basovizza, 34149 Trieste, Italy. \\ ${ }^{b}$ Department of Physics, University of Trieste, 34128 Trieste, Italy. \\ ${ }^{c}$ IOM CNR, Laboratorio TASC, Area Science Park Basovizza, 34149 Trieste, Italy. \\ ${ }^{d}$ Istituto Nazionale di Fisica Nucleare, INFN Sezione di Trieste, Trieste, 34100, Italy. \\ ${ }^{e}$ Department of Medical Imaging, University of Saskatchewan, Saskatoon, SK S7N 5A2, Canada. \\ ${ }^{f}$ DPIA, University of Udine, Via delle Scienze 206, 33100 Udine, Italy. \\ ${ }^{g}$ DIEF, University of Modena and Reggio Emilia, Via Trovarelli 2, 44100 Modena, Italy. \\ E-mail: camilla.nichetti@elettra.eu
}

Aвstract: Avalanche photodiodes based on GaAs/AlGaAs with separated absorption and multiplication regions (SAM-APDs) will be discussed in terms of capacitance, response to light (gain and noise) and time response. The structures have been fabricated by molecular beam epitaxy introducing a $\delta \mathrm{p}$ layer doped with carbon to separate the multiplication and the absorption regions. The thickness of the latter layer defines the detection efficiency and the time resolution of the structure, which in turn allows tailoring the device for specific scientific applications. Within the multiplication region a periodic modulation of the bandgap is obtained by growing alternating nanometric layers of $\mathrm{AlGaAs}$ and $\mathrm{GaAs}$ with increasing $\mathrm{Al}$ content; this staircase structure enables the tuning of the bandgap and subsequently provides a well-defined charge multiplication. The use of such staircase hetero-junctions enhances electron multiplication and conversely reduces - at least in principle - the impact of the noise associated to hole multiplication, which should result in a decreased overall noise, when compared to $\mathrm{p}-\mathrm{i}-\mathrm{n}$ diodes composed by a single material. The first part of this paper focuses on the electrical characteristics of the grown structure and on the comparison with the simulated behaviour of such devices. In addition, gain and noise measurements, which have been carried out on these devices by utilizing photons from visible light to hard X-rays, will be discussed and will be compared to the results of a nonlocal history-dependent model specifically developed for staircase APDs.

KeYwords: GaAs, avalanche photodiode, noise, time response

ArXIV ePrINT: 1234.56789

${ }^{1}$ Corresponding author. 


\section{Contents}

1 Introduction 1

2 APD design 2

3 Experimental results 3

3.1 Capacitance as a function of the applied bias 3

3.2 Current maps 3

3.3 Gain and noise analysis 4

3.4 Time response measurements 6

4 Conclusion $\quad 7$

\section{Introduction}

Materials such as GaAs can be of particular interest for applications as detectors exposed to high energy radiation. In fact, GaAs detectors have a number of advantages over narrow bandgap semiconductor materials, such as Si. The relatively wide bandgap of GaAs $(1.42 \mathrm{eV}$ at $300 \mathrm{~K})$ makes the number of thermally generated carriers lower than that of narrow bandgap materials, resulting in a lower leakage current which allows X-rays to be detected even at room temperature [1]. Another advantage of GaAs is its relatively low electron-hole pair creation energy $(4.184 \mathrm{eV}$ at $300 \mathrm{~K})$ [2]. Moreover, the high effective atomic number of GaAs results in a much higher absorption coefficient [3] and provides higher detection efficiency than a Si sensor of the same thickness [1]. This could also be seen from a different point of view: having a shorter absorption length would allow having a thinner device, which combined with a larger electron mobility could translate into shorter response times.

For these reasons, $\mathrm{Al}_{\mathrm{x}} \mathrm{Ga}_{1-\mathrm{x}} \mathrm{As}$ alloys are going to be a suitable material for radiation detection in environments which suffer from high radiation doses, such as synchrotron beamline, in principle without the need of a cooling system [1]. However, III-V semiconductors feature very similar electron and hole ionization coefficients leading to higher multiplication noise [4]. To overcome this problem, superlattice structures have been suggested in the literature [5]. A staircase structure based on superlattices has been incorporated in the separated absorption and multiplication avalanche photodiodes which are here reported. The structure profits from the use of bandgap engineering which artificially enables to increase the difference between the effective ionization coefficients of holes and electrons, introducing conduction band discontinuities that provide energy to the moving electrons and valence band steps that subtract energy from moving holes. The devices under discussion were grown by molecular beam epitaxy (MBE) and fabricated at the CNR-IOM laboratory and eventually tested at the Elettra synchrotron facility in Trieste. 
Table 1. Layer details of the SAM-APD structure from which the devices were fabricated.

\begin{tabular}{lccccc}
\hline Material & Dopant & Dopant type & Thickness $(\mathrm{nm})$ & Doping density & Repetitions \\
\hline $\mathrm{GaAs}$ & $\mathrm{C}$ & $\mathrm{p}$ & 150 & $6 \times 10^{18} \mathrm{~cm}^{-3}$ & \\
$\mathrm{GaAs}$ & & & 4500 & & \\
$\delta$ layer & $\mathrm{C}$ & $\mathrm{p}$ & & $2.5 \times 10^{12} \mathrm{~cm}^{-2}$ & \\
$\mathrm{GaAs}$ & & & 35 & & \\
$\mathrm{Al}_{\mathrm{x}} \mathrm{Ga}_{1-\mathrm{x}} \mathrm{As}$ & & & 20 & & \\
$\mathrm{AlGaAs}$ & & & 25 & & \\
$\mathrm{GaAs}$ & & & 35 & & \\
$\mathrm{GaAs}$ & $\mathrm{Si}$ & $\mathrm{n}$ & 100 & $2 \times 10^{18} \mathrm{~cm}^{-3}$ & \\
\hline
\end{tabular}

\section{APD design}

The APDs made of GaAs/AlGaAs epitaxial layers were grown by MBE on a 500- $\mu$ m-thick heavily Si-doped n-type GaAs substrate. The resulting layered structure is summarized in Table 1 and Figure 1 [6]. Mesa diodes with different diameters $(200 \mu \mathrm{m}$ and $600 \mu \mathrm{m})$ were chemically etched by using the solution $\mathrm{H}_{3} \mathrm{PO}_{4}: \mathrm{H}_{2} \mathrm{O}_{2}: \mathrm{H}_{2} \mathrm{O}$ (3:1:50). The metallization of the p-contact consists of a layer of $10 \mathrm{~nm}$ of $\mathrm{Cr}$ followed by $50 \mathrm{~nm}$ of Au. This top contact covers the $75 \%$ of the surface of the $200-$ $\mu \mathrm{m}$ diameter devices and the $58 \%$ of the $600-\mu \mathrm{m}$ ones. The rear contact, instead, consists of $50 \mathrm{~nm}$ of $\mathrm{AuGe}, 10 \mathrm{~nm}$ of $\mathrm{Ni}$ and $40 \mathrm{~nm}$ of Au. Furthermore, to reduce the leakage currents, the devices were passivated with $\mathrm{SiO}_{2}$, grown by sputtering techniques. Another important aspect of this structure is the $\delta$ p-doped layer created by a submonolayer of carbon atoms. Since its dose controls the separation between the absorption and the multiplication regions and up to which voltage this is preserved, several devices with different carbon concentrations have been fabricated and have been characterized [6]. The minimum dose to achieve complete depletion of the multiplication region $\left(2.5 \times 10^{12} \mathrm{~cm}^{-2}[6]\right)$ was used to fabricated the devices presented here.

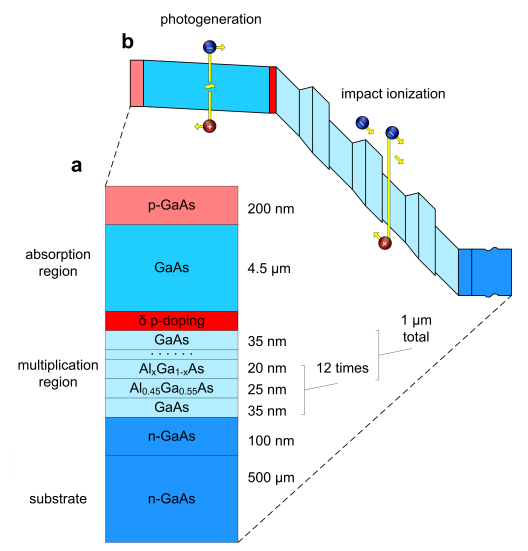

Figure 1. Layer sequence of the SAM-APD device structure (a) and band profiles under an applied bias (b).

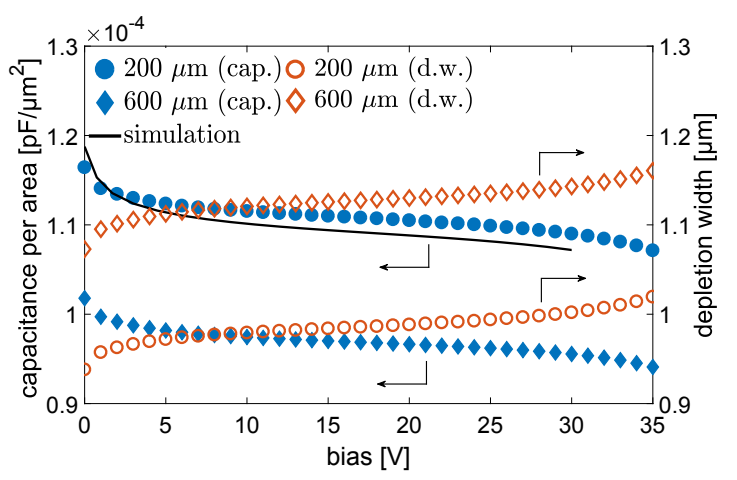

Figure 2. Capacitance and depletion width as functions of the reverse applied bias. 


\section{Experimental results}

The electrical characterization of the devices was performed under different conditions: in dark, under a green laser and under hard X-rays, always at room temperature.

\subsection{Capacitance as a function of the applied bias}

The capacitances, measured as a function of applied reverse bias through a precision frequency LCR meter (HP4284A), made it possible to determine the depletion width and the doping concentration inside the devices. The test signal was sinusoidal with $30 \mathrm{mV}$ rms magnitude and set at two different frequencies, $400 \mathrm{kHz}$ and $1 \mathrm{MHz}$, respectively. These measurements were carried out at reverse voltages between $0 \mathrm{~V}$ and $35 \mathrm{~V}$. Since there was no significant difference between the measurements taken at the two frequencies above, only the data obtained at $1 \mathrm{MHz}$ are reported here. Moreover, all the capacitances didn't drift apart more than $5 \%$ with respect to the expected capacitance value corresponding to the depletion width of the multiplication region (i.e. 1- $\mu \mathrm{m}$ ). The width of the depletion layer of the diodes as a function of the applied reverse voltage was computed by using the measured capacitance [7] as

$$
d(V)=\frac{\varepsilon_{r} \varepsilon_{0} A}{C(V)}
$$

where $A$ is the area of the mesa, $C$ is the measured capacitance, $\varepsilon_{r}=12.6[8]$ and $\varepsilon_{0}=8.854 \times 10^{-12}$ $\mathrm{F} / \mathrm{m}$ are the equivalent relative permittivity of the active region (calculated as the average value of the permittivity of the different materials in the depletion region) and the permittivity of vacuum, respectively. Figure 2 shows the capacitance characteristics of two photodiodes in blue, $200 \mu \mathrm{m}$ (full circles) and $600 \mu \mathrm{m}$ (full diamonds) in diameter, performed at $1 \mathrm{MHz}$ and their relative depletion widths in red, (empty circles and empty diamonds, respectively) calculated by using Equation 3.1. The black line indicates the values obtained with the TCAD [9] simulation of the 1D section of the structure under test.

As a further analysis, starting from the capacitance, it is possible to calculate the profile of dopant concentration inside the device, $N(d)$, by using the equation for general nonuniform distributions [7]

$$
\frac{d\left(1 / C_{A}^{2}\right)}{d V}=\frac{2}{q \varepsilon_{r} \varepsilon_{0} N(d)}
$$

where $C_{A}$ is the capacitance per unit of area of the mesa, $V$ is the applied bias and $q$ is the elementary charge. The results of this extraction can be seen in Figure 3, where the dopant concentration is plotted as a function of the depletion width: the blue dots represent the values obtained with the measured capacitances, while the red line shows the results obtained with a polynomial fit of the inverse of the capacitance optimized with the least squares method, both by using Equation 3.2. It is possible to compare the area under the curve with the dose of carbon atoms which is nominally insert in this layer $\left(2.5 \times 10^{12} \mathrm{~cm}^{-2}\right)$. The areas have a value of $2.4 \times 10^{12} \mathrm{~cm}^{-2}$ and $2.1 \times 10^{12} \mathrm{~cm}^{-2}$ calculated from Figure 3a and Figure 3b, respectively.

\subsection{Current maps}

To assess the response to photons of different energies, the devices were tested under hard X-rays at the XRD2 beamline [10] and soft X-rays at the Circular Polarization beamline (CiPo) [11] of Elettra. 


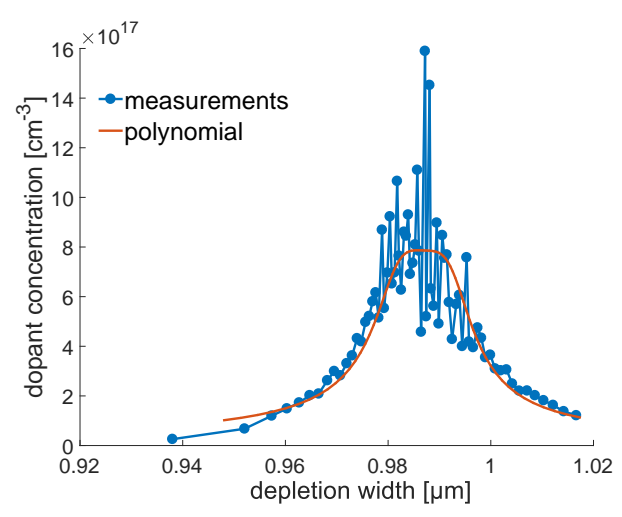

(a)

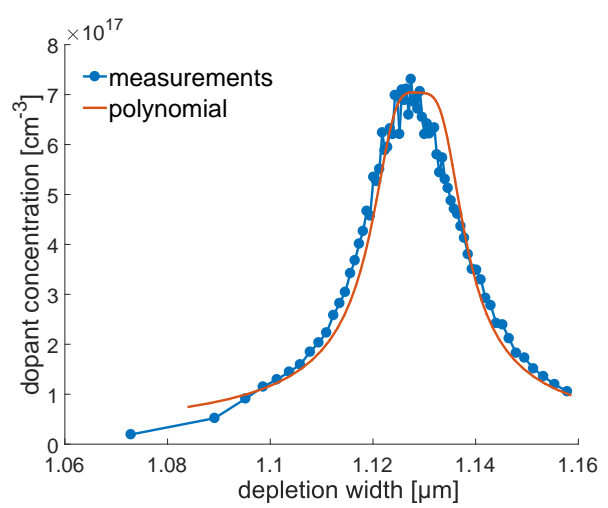

(b)

Figure 3. Dopant concentration as function of depletion width for two devices characterized by having diameters of $200 \mu \mathrm{m}$ (a) and of $600 \mu \mathrm{m}$ (b).

In particular, some current maps were acquired using different bias voltages. Figure 4 shows the maps specifically obtained with $12.4-\mathrm{keV}$ photons at the XRD2 beamline, depicted as surfaces. The one measured at $5 \mathrm{~V}$ (surface below) shows how the charge collection is low in the centre of the mesa with respect to its edge: the lowest value is $50 \%$ of the maximum value and the transition is quite smooth, meaning that the electric field established under this bias in the central part of the mesa is not high enough to drive the electrons created in that part down to the multiplication region before they recombine. Applying $31 \mathrm{~V}$ (avalanche region) the photocurrent surface appears flatter and more uniform, and the lowest value acquired is $80 \%$ of the maximum value, which shows that under this bias the field is already able (because of both its higher absolute values and its increased uniformity) to drive a large part of the electrons created in the centre of the mesa down to the multiplication region. The overall lower current in the central part of the mesa is due to the higher absorption of the gold contact.

\subsection{Gain and noise analysis}

The devices were tested with different photon energies to asses their response to light. A thermostated green tabletop laser $(\lambda=532 \mathrm{~nm})$ and soft $(500 \mathrm{eV}-800 \mathrm{eV}$ range $)$ and hard X-ray $(12.4 \mathrm{keV})$ sources were used. The obtained gain trend looks similar for all the different energies utilized to generate the photo-currents and the gain itself is defined as

$$
M=I_{p h} / I_{p h o}
$$

where $I_{p h}$ is the difference between the measured photocurrent and the current measured under dark conditions and $I_{p h o}$ is $I_{p h}$ at unity gain. Therefore, in Figure 5 we present the currents (and the corresponding gains) acquired through a 4-channel picoammeter (AH501 by Elettra) while deliberately changing the impinging flux by means of different $\mathrm{Al}$ absorbers at the XRD2 beamline. The gains are almost coincident for the different photon fluxes of the hard X-rays, showing how even at high energies the behaviour follows the expected one. However, it must be pointed out that these values are slightly lower than what is usually obtained under ideal injection conditions, that 


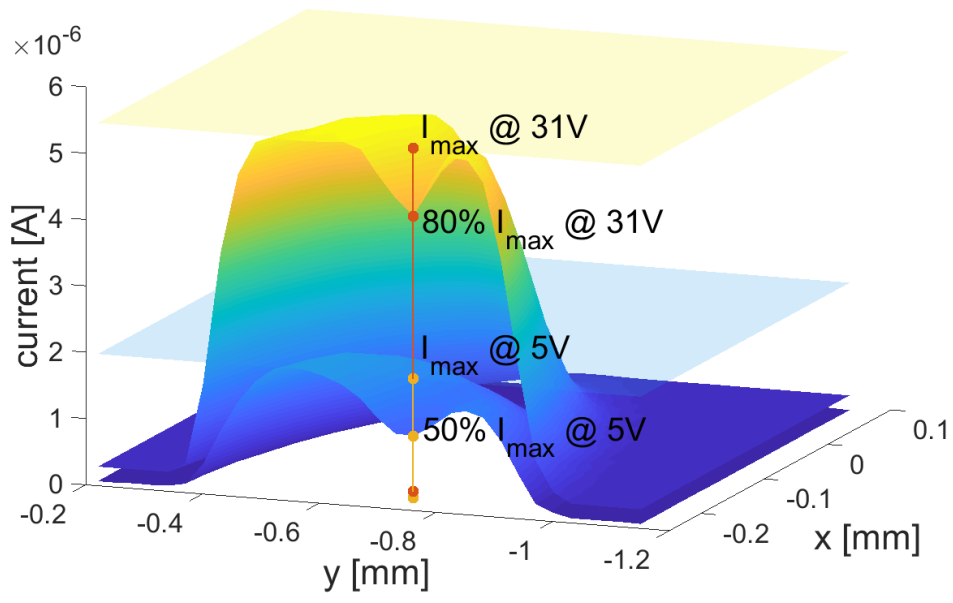

Figure 4. Current maps at $5 \mathrm{~V}$ (surface below) and $31 \mathrm{~V}$ (surface above) for the device with a mesa diameter of $600 \mu \mathrm{m}$. The currents outside the mesa are due to the sum of the dark current with a residual response of the die caused by tails of the beam.

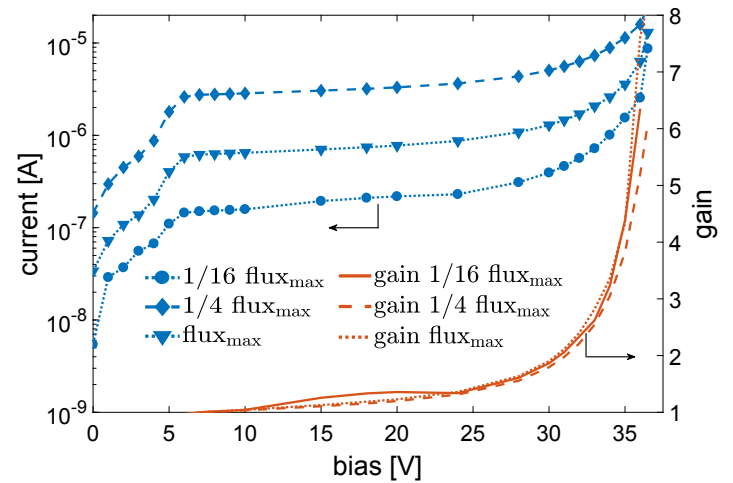

Figure 5. Currents and gains as a function of the applied reverse bias measured at the XRD2 beamline. The maximum flux here reported was obtained adjusting the $\mathrm{Al}$ absorber so that only $4 \%$ of the highest flux provided by the beamline was transmitted.

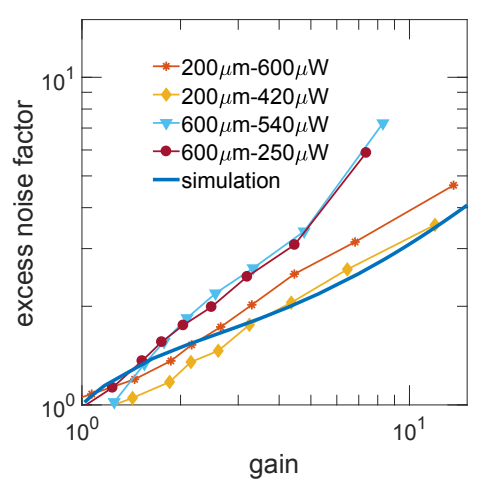

Figure 6. Excess noise factor as a function of the gain extracted from measurements performed with a thermostated green laser: red circle, 250 $\mu \mathrm{W}$, light blue triangle, $540 \mu \mathrm{W}$ for the devices with $600 \mu \mathrm{m}$ in diameter; yellow diamonds, 420 $\mu \mathrm{W}$, orange stars, $600 \mu \mathrm{W}$ for the devices with $200 \mu \mathrm{m}$ in diameter.

is when electron and hole pairs are created just in the absorption region, not in the entire structure as when using higher photon energies.

The current and the noise measured while the device was illuminated by the laser were used to calculate the excess noise factor $F$, which can be defined as the ratio between the power spectral density of the photogenerated current $\left(S_{i i, p h}\right)$ and the power spectral density of the shot noise $\left(S_{i i, s h o t}\right)$ multiplied by the square of the gain: 


$$
F=\frac{S_{i i, p h}}{S_{i i, s h o t} \cdot M^{2}} .
$$

Eventually, as $M$ and $F$ are strictly related to one another, they were consistently scaled in order for $F$ to be one at unity gain. As a validation for the excess noise factor estimations obtained through the laser measurements, the resulting values of $F(M)$ were compared with the ones obtained by simulating the structure with the Energy Balance History- Dependent Model (EBHDM) presented in [12], with the nominal device parameters as in [13]. Figure 6 compares these results by plotting $F$ as a function of $M$. We see that the values obtained with the setup described in [14] (symbols) follow the trend suggested by the EBHDM simulations (purple curve) fairly well and, in particular, for a specific diameter they are very consistent with each other even at different laser powers. The higher excess noise factor for the same gain for the large diameter devices is probably due to a larger influence of the substrate, however further investigations are needed.

\subsection{Time response measurements}

Some preliminary timing measurements were performed by taking advantage of the bunch structure of Elettra. Although the experimental setup has not been optimized for precise timing measurements, the acquired data allows us to extract some quantitative metrics for the APD response.

The synchrotron facility was operating in its normal filling mode, providing 432 equidistant bunches per revolution (each bunch is characterized by a length of approximately $150 \mathrm{ps}$ and outdistanced from the following one by $2 \mathrm{~ns}$ ) and including a dark gap, which was used as trigger for the oscilloscope (KEYSIGHT DSOS404A) featuring a bandwidth of $4 \mathrm{GHz}$, a sample rate of 20 $\mathrm{GSa} \cdot \mathrm{s}^{-1}$ and a 10-bit resolution. The characteristic impedance of the impedance-matched coaxial cabling, which connects the device to the oscilloscope, in combination with the capacitance of the device turns into a low-pass filter with a time constant of approximately $1.5 \mathrm{~ns}$, which acts as an integrator for the substantially shorter bunch signals. Figure 7 a shows the traces acquired by the oscilloscope for different bias voltages utilized during these measurements. From this image it can be seen that as the bias increases the signals show the intrinsic gain of the structure.

According to these considerations, the measured bunch signal should be the integrated convolution of the photon bunch $(b(t)$, which is a pulse train representing the photon bunches delivered by Elettra) with the time response $(r(t))$ of the APD. To obtain the convolved signal $(m(t)=b(t) * r(t))$ of the bunch with the APD time response, the low-pass filtered signal (Figure 7a) was firstly derived in time to compensate for the low-pass nature of the $\mathrm{RC}$ due to the cable and the detector. Then, to extract the time response of the APD it was necessary to deconvolve $b(t)$ out of $m(t)$, or alternatively, operating in the frequency domain

$$
r(t)=\left|\mathfrak{F}^{-1}\left[\frac{\mathfrak{F}(m(t))}{\mathfrak{F}(b(t))}\right]\right|^{2}
$$

where $\mathfrak{F}$ is the Fourier transform. The result of the deconvolution $(r(t))$ is shown in Figure $7 \mathrm{~b}$. The diamonds are the deconvolved smoothed data points while the solid line is the exponentially modified Gaussian (EMG) distribution, which describes the sum of independent normal and exponential random variables, in other words a convolution of the normal and the exponential distribution. The Gaussian component is characterized by mean $\mu$ and variance $\sigma^{2}$, and the exponential one by rate 
$\lambda$. It has a characteristic positive skew from the exponential component, which is the expected signal from an APD [15]. In this case the fit parameters can be quoted with $\mu=4.62 \times 10^{-10} \mathrm{~s}, \sigma$ $=14.3 \mathrm{ps}$, height $4.1 \times 10^{-4}$ and $\lambda=1 / \tau$ with $\tau=66 \mathrm{ps}$ resulting in a full width at half maximum (FWHM) of $r(t)$ of about $100 \mathrm{ps}$ and a rise time of $25 \mathrm{ps}$.

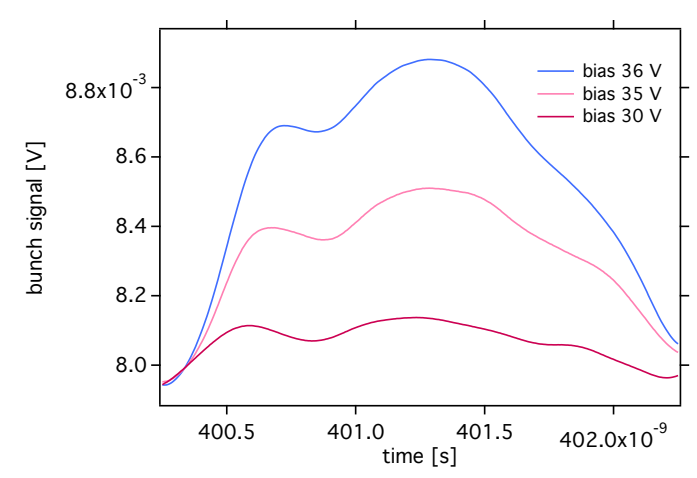

(a)

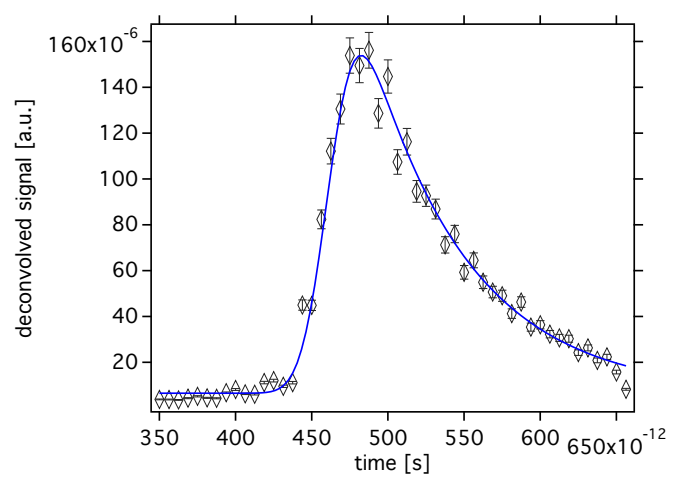

(b)

Figure 7. (a) Time traces acquired at the XRD2 beamline by the oscilloscope for different bias voltages. (b) Time response of the APD $(R(t))$.

\section{Conclusion}

The SAM-APDs were electrically characterized, by analysing the carrier profile of the $\delta$ layer, and tested under photons of different energies to asses their response to light. The currents and the power spectra densities were then used to calculate the avalanche gain and the excess noise factor. The results are in excellent agreement with the values of the simulation and they are consistent between devices of different diameters characterized at various photon fluxes. The time response of the APD can be interpreted as an exponentially modified Gaussian distribution with a FWHM of about $100 \mathrm{ps}$ and a rise time of $25 \mathrm{ps}$.

\section{Acknowledgments}

The research in this work received funding by the Italian MIUR through the PRIN 2015 project 2015WMZ5C8. Furthermore, the authors would like to thank M. Danailov, G. Brajnik, G. Bais, A. Heroux, D.G. Dumitrescu and G. Lautizi for their support during the experiments.

\section{References}

[1] G. Lioliou, X. Meng, J. Ng, and A.M. Barnett. Characterization of Gallium Arsenide X-ray mesa p-i-n photodiodes at room temperature. Nucl. Instr. and Meth. A, 813:1-9, 032016.

[2] G. Lioliou and A.M. Barnett. Gallium Arsenide detectors for X-ray and electron (beta particle) spectroscopy. Nucl. Instr. and Meth. A, 836:37-45, 082016.

[3] J.H. Hubbell. Photon mass attenuation and energy-absorption. Int. J. Appl. Radiat. Isot., 33:1269-1290, 1982. 
[4] R.J. McIntyre. A new look at impact ionization-part I: A theory of gain, noise, breakdown probability, and frequency response. IEEE Electr. Device Lett., 46:1623 - 1631, 1999.

[5] F. Capasso, W.T. Tsang, and G. Williams. Staircase Solid-State Photomultipliers and Avalanche Photodiodes with Enhanced Ionization Rates Ratio. IEEE Trans. Electron. Devices, 30:381 - 390, 05 1983.

[6] T. Steinhartova, C. Nichetti, M. Antonelli, G. Cautero, R.H. Menk, A. Pilotto, F. Driussi, P. Palestri, L. Selmi, K. Koshmak, S. Nannarone, F. Arfelli, S. Dal Zilio, and G. Biasiol. Influence of p-doping on the behaviour of AlGaAs/GaAs SAM-APDs for synchrotron radiation. J. Instrum., 12:C11017, 2017.

[7] S.M. Sze. Physics of Semiconductor Devices. John Wiley \& Sons, 1981.

[8] M. Levinshtein and S. Rumyantsev. Handbook Series on Semiconductor Parameters, volume 1. 1996.

[9] Synopsys, sentaurus ${ }^{\mathrm{TM}}$ device user guide, 2006.

[10] XRD2. Elettra and FERMI lightsources, 2018.

[11] CiPo. Elettra and FERMI lightsources, 2018.

[12] C. Nichetti, A. Pilotto, P. Palestri, L. Selmi, M. Antonelli, F. Arfelli, G. Biasiol, G. Cautero, F. Driussi, Y.N. Klein, R. H. Menk, and T. Steinhartova. An Improved Nonlocal History-Dependent Model for Gain and Noise in Avalanche Photodiodes Based on Energy Balance Equation. IEEE T. Electron Dev., 65:1823-1829, 2018.

[13] J. Lauter, A. Forster, H. Luth, K.D. Muller, and R. Reinartz. AlGaAs/GaAs avalanche detector array-1 GBit/s X-ray receiver for timing measurements. IEEE Trans. Nucl. Sci., 43:1446 - 1451, 1996.

[14] C. Nichetti, T. Steinhartova, M. Antonelli, G. Cautero, R. H. Menk, A. Pilotto, F Driussi, P. Palestri, L. Selmi, F. Arfelli, and Biasiol. Investigation of the behaviour of AlGaAs/GaAs SAM-APDs for synchrotron radiation. AIP Conf. Proc., 2018.

[15] K. Zang, X. Jiang, Y. Huo, X. Ding, M. Morea, X. Chen, C.Y. Lu, J. Ma, M. Zhou, Z. Xia, Z. Yu, I.T. Kamins, Q. Zhang, and J. Harris. Silicon single-photon avalanche diodes with nano-structured light trapping. Nat. Commun., 8, 122017. 